\title{
The effect of changes in intraocular pressure on the risk of primary open-angle glaucoma in patients with ocular hypertension: an application of latent class analysis
}

\author{
Feng Gao ${ }^{1 *}$, J Philip Miller', Stefano Miglior ${ }^{3}$, Julia A Beiser ${ }^{2}$, Valter Torri ${ }^{4}$, Michael A Kass ${ }^{2}$ and Mae O Gordon ${ }^{1,2}$
}

\begin{abstract}
Background: Primary open-angle glaucoma (POAG) is one of the leading causes of blindness in the United States and worldwide. While lowering intraocular pressure (IOP) has been proven to be effective in delaying or preventing the onset of POAG in many large-scale prospective studies, one of the recent hot topics in glaucoma research is the effect of IOP fluctuation (IOP lability) on the risk of developing POAG in treated and untreated subjects.

Method: In this paper, we analyzed data from the Ocular Hypertension Treatment Study (OHTS) and the European Glaucoma Prevention Study (EGPS) for subjects who had at least 2 IOP measurements after randomization prior to POAG diagnosis. We assessed the interrelationships among the baseline covariates, the changes of post-randomization IOP over time, and the risk of developing POAG, using a latent class analysis (LCA) which allows us to identify distinct patterns (latent classes) of IOP trajectories.

Result: The IOP change in OHTS was best described by 6 latent classes differentiated primarily by the mean IOP levels during follow-up. Subjects with high post-randomization mean IOP level and/or large variability were more likely to develop POAG. Five baseline factors were found to be significantly predictive of the IOP classification in OHTS: treatment assignment, baseline IOP, gender, race, and history of hypertension. In separate analyses of EGPS, LCA identified different patterns of IOP change from those in OHTS, but confirmed that subjects with high mean level and large variability were at high risk to develop POAG.
\end{abstract}

Conclusion: LCA provides a useful tool to assess the impact of post-randomization IOP level and fluctuation on the risk of developing POAG in patients with ocular hypertension. The incorporation of post-randomization IOP can improve the overall predictive ability of the original model that included only baseline risk factors.

Keywords: Latent class analysis, Longitudinal data, Time-dependent covariate, Prediction model, Survival data, Primary open-angle glaucoma, Intraocular pressure fluctuation

\section{Background}

Ocular hypertension is a leading risk factor for the development of primary open-angle glaucoma (POAG) which remains one of the major causes of blindness in the United States and worldwide [1-5]. It is estimated that approximately $4 \%-7 \%$ of the population over the age of 40 years have ocular hypertension without detectable glaucomatous damage using standard clinical

\footnotetext{
*Correspondence: feng@wustl.edu

'Division of Biostatistics, Washington University School of Medicine, St. Louis, MO 63110, USA

Full list of author information is available at the end of the article
}

tests, and thus as many as 3 to 6 million Americans are at risk for developing glaucoma because of ocular hypertension [6-8]. Intraocular pressure (IOP) is the only known modifiable risk factor for POAG. Lowering the level of IOP has been shown to effectively delay or prevent glaucomatous visual damage in different phases of disease progression by many large-scale multicenter clinical trials, including the Ocular Hypertension Treatment Study (OHTS) [9], the Early Manifest Glaucoma Trial [10], and the Advanced Glaucoma Intervention Study [11]. 
In recent years, one of the hot topics in glaucoma research has been the effect of IOP fluctuation (IOP lability), both within a single day (short-term fluctuation) and from visit to visit (long-term fluctuation) on POAG [12,13]. Measures of IOP fluctuation have included a wide range of quantities - peak, trough, variance, and range, etc. [13] However, since subjects with high mean IOP often show large IOP variability over time, it is challenging to disentangle the effect of fluctuation from mean IOP. A recently emerged technique for longitudinal data analysis, latent class analysis (LCA) [14], provides an appealing approach to this question. Rather than dealing with individual measures of fluctuation, LCA identifies distinct patterns of longitudinal profiles based on the combination of summary statistics (i.e., mean level and variability) and hence provides information complementary to the conventional methods. LCA uses the patterns of serial biomarker readings available for subjects, together with baseline covariates and disease outcomes, to divide subjects into a number of mutually exclusive subpopulations (classes). The class membership is unobserved (latent) and determined by the class-specific parameters in a data-driven basis.

In this paper, we used LCA to model the postrandomization IOP in the OHTS. For each class, the change of IOP was characterized by 4 parameters: the initial IOP level (I), the linear (L) and quadratic (Q) trend over time, and the variance of IOP (V). We used data from the European Glaucoma Prevention Study (EGPS) [15], another large-scale multicenter randomized clinical trial of patients with ocular hypertension, for external independent validation. We first fit an unconditional (without any covariates) LCA to determine the optimal number of distinct patterns that best described the IOP change for each study. Then a conditional model was constructed by adding baseline covariates as the antecedents (predictors) of IOP change and time to POAG as a consequence (outcome) of IOP change [16]. This analysis enhanced our understanding of the interrelationships among the IOP change, the baseline covariates, and the risk of developing POAG. This also provided evidence towards our ultimate goal to improve the prediction of POAG in patients with ocular hypertension.

\section{Methods}

\section{Study cohort}

Our study used data from OHTS and EGPS, the two largest randomized trials to test safety and efficacy of topical hypotensive medication in preventing the development of POAG. In OHTS, 1636 subjects were randomized to either observation or treatment with ocular hypotensive medication and followed for a median of
78 months [9]. In EGPS, 1077 subjects were randomized to either placebo or an active treatment (dorzolamide) and followed for a median of 55 months [15]. The two studies shared many key similarities in the study protocol and generated data of high quality. In both studies, for example, the outcome ascertainment was performed by specialized resource centers where readers were masked as to randomization assignment and information about the participant's clinical status, and the attribution of abnormality due to POAG was performed by a masked Endpoint Committee. Detailed information on the similarity and discrepancy between OHTS and EGPS as described by Gordon et al. [17]. This study was approved by the Institutional Review Boards of Washington University in St. Louis and the University Bicocca of Milan.

In this paper, we excluded IOP values measured after POAG onset. The primary endpoint was time from randomization to the development of POAG. Those subjects who did not develop POAG were censored at the date of study closeout. In addition to the follow-up data, following 13 demographic and clinical characteristics at randomization were also included in this paper: treatment assignment (TRT, 0 for observation/placebo and 1 for treatment), male gender (Male), black race (Black), age at randomization (Age, decade), baseline IOP (IOP0, $\mathrm{mmHg}$ ), central corneal thickness $(\mathrm{CCT}, \mu \mathrm{m})$, pattern standard deviation (PSD, $\mathrm{dB}$ ), vertical cup/disc ratio (VCD), the use of systematic beta blocker (BB) or Calcium channel blockers $(\mathrm{CHB})$, and the history of diabetes (DM), heart diseases (Heart), or hypertension (HBP). These baseline factors were identified a priori as possible predictors for the development of POAG during the planning phase of the OHTS [18]. We excluded 34 subjects from EGPS with pigment dispersion and exfoliation syndromes (an exclusion criterion in OHTS). We also excluded subjects without any follow-up data (18 in OHTS and 47 in EGPS) or those with only 1 follow-up visit (19 in OHTS and 25 in EGPS). Therefore, these subjects with at least 2 follow-up visits (1600 from OHTS and 971 from EGPS) constituted our study cohort for the unconditional LCA. In the conditional LCA, we further excluded subjects without CCT measurements (169 in OHTS and 143 in EGPS) or those with missing values in any other baseline factors (6 in EGPS). Table 1 presented the summary statistics of baseline covariates and post-randomization data for each study, where the binary data were summarized as counts and proportions, while the continuous variables were summarized in means and standard deviations (SD). For consistency with previous analyses $[17,18]$, values for the baseline eye-specific variables (CCT, PSD, VCD, and baseline IOP) for each participant were the average of two eyes (with the exception of the EGPS participants 


\begin{tabular}{|c|c|c|}
\hline Variables & OHTS $(\mathrm{N}=1600)$ & EGPS $(N=971)$ \\
\hline \multicolumn{3}{|l|}{ Baseline predictors } \\
\hline TRT & $795(49.7 \%)$ & $487(50.2 \%)$ \\
\hline Male & $687(42.9 \%)$ & $445(45.8 \%)$ \\
\hline Black & $396(24.8 \%)$ & $1(0.1 \%)$ \\
\hline AGE (decades) & $5.56(0.96)$ & $5.70(1.02)$ \\
\hline IOPO (mmHg) & $24.9(2.69)$ & $23.4(1.62)$ \\
\hline $\mathrm{CCT}(\mu \mathrm{m})$ & $572.6(38.5)$ & $573.3(37.5)$ \\
\hline PSD (dB) & $1.91(0.21)$ & $2.00(0.52)$ \\
\hline VCD & $0.39(0.19)$ & $0.32(0.14)$ \\
\hline BB & $71(4.4 \%)$ & $64(6.6 \%)$ \\
\hline $\mathrm{CHB}$ & $190(11.9 \%)$ & $66(6.8 \%)$ \\
\hline $\mathrm{DM}$ & $188(11.8 \%)$ & $55(5.7 \%)$ \\
\hline Heart & $99(6.2 \%)$ & $109(11.2 \%)$ \\
\hline HBP & $606(37.9 \%)$ & $279(28.7 \%)$ \\
\hline \multicolumn{3}{|l|}{ Post-randomization IOP } \\
\hline Mean $(\mathrm{mmHg})$ & $21.44(3.45)$ & $19.73(2.57)$ \\
\hline $\mathrm{SD}(\mathrm{mmHg})$ & $2.27(1.04)$ & $2.22(1.03)$ \\
\hline Median \#visits (min-max) & $13(2-16)$ & $9(2-10)$ \\
\hline POAG & $146(9.1 \%)$ & $107(11.0 \%)$ \\
\hline
\end{tabular}

with only one eye eligible for the study). For the post-randomization IOP, however, only eye-specific data were used because averaging two eyes could underestimate the true intra-patient IOP variability. We took advantage of the fact that IOPs between two eyes were highly correlated (with an intra-class correlation coefficient of 0.75), and follow-up IOPs were chosen from the first eye developed POAG or an eye selected randomly in participants without POAG. Since the continuous baseline covariates were measured in quite different scales, they were standardized to have mean 0 and variance 1 throughout the remainder of this paper. As such, for these variables the odds ratios (OR) and hazard ratios (HR) from the regression models represented the effect per 1-SD change.

\section{Statistical analysis} Unconditional LCA

Suppose there were $N$ subjects and each subject had $n_{i}$ pre-POAG IOP measures. Let $Y_{i}=\{Y 1, Y 2, \ldots \ldots$.$\} denote$ the post-randomization IOP and $\mathrm{C}_{i}$ represent the latent class membership of $i^{\text {th }}$ individual, and $\theta_{\mathrm{g}}$ be the vector of class-specific parameters that differentiate the G latent classes, with $i=1,2, \ldots, N$, and $g=1,2, \ldots, G$, respectively. Then the distribution of $Y_{i}$ was a mixture distribution defined as [14],

$$
f\left(Y_{i}\right)=\sum_{g=1}^{G}\left\{\operatorname{Pr}\left(C_{i}=g\right) \bullet f\left(Y_{i} \mid C_{i}=g ; \theta_{g}\right)\right\}
$$

where $\operatorname{Pr}\left(C_{i}=g\right)$ represented the size (mixing proportion) of $g^{\text {th }}$ latent class in the mixture and $f\left(Y_{i} \mid C_{i}=g ; \theta_{g}\right)$ was the class-specific distribution of $Y_{i}$ as detailed below.

- The mixing probability $\operatorname{Pr}\left(C_{i}=g\right)$ was modeled as a multinomial logistic regression, $\operatorname{Pr}\left(C_{i}=g\right)=$ $\frac{\exp \left(\alpha_{0 g}\right)}{\sum_{h=1}^{G} \exp \left(\alpha_{0 g}\right)}$, where $\alpha_{0 g}$ represented the log odds of membership in the $g^{\text {th }}$ class relative to a reference class (class 1, say), with the parameter in the reference being 0 for identification.

- The specification of $f\left(Y_{i} \mid C_{i}=g ; \theta_{g}\right)$ was aided by our previous experience on the joint modeling of longitudinal IOP and time to POAG in OHTS [19]. The joint model identified IOP variability as an independent predictor for POAG and also revealed that the IOP change can be better fit by a quadratic functional form. Therefore, we set $f\left(Y_{i} \mid C_{i}=g ; \theta_{g}\right)=I_{g}+L_{g} t_{i}+Q_{g} t_{i}^{2}+\epsilon_{i}$, with $\epsilon_{i}^{\sim} \mathrm{N}\left(0, \mathrm{~V}_{\mathrm{g}}\right)$ and $\theta_{g}=\left\{I_{g}, L_{g}, Q_{g}, \mathrm{~V}_{\mathrm{g}}\right\}$. Because high IOP was an eligibility criterion in both OHTS and EGPS, the estimated initial level (intercept $I_{\mathrm{g}}$ ) may be influenced by "regression to the mean". To address this concern, we re-set the time 0 and the intercept was actually estimated at 1-year after randomization. We also assumed that follow-up IOPs were measured regularly every 6 months according to the protocol, i.e., with timing $t_{i}=\{-0.5,0,0.5,1, \ldots\}$. Figure 1A showed the diagram of an unconditional LCA for the OHTS data.

- Given the estimated parameters $\widehat{\theta} g$ and the observed IOP, each individual can be assigned to the most likely class based on the probability of class membership (often termed as posterior class probability) [14],

$$
\text { - } p_{i g}=\frac{\widehat{\operatorname{Pr}}\left(C_{i}=g\right) \bullet f\left(Y_{i} \mid C_{i}=g ; \widehat{\theta}_{g}\right)}{\sum_{h=1}^{G}\left\{\widehat{\operatorname{Pr}}\left(C_{i}=h\right) \bullet f\left(Y_{i} \mid C_{i}=h ; \widehat{\theta}_{h}\right)\right\} .}
$$

The best unconditional LCA was selected by enumerating and comparing a set of competing models differing only in the number of classes. In this paper, the model comparison was based primarily on the log likelihood 


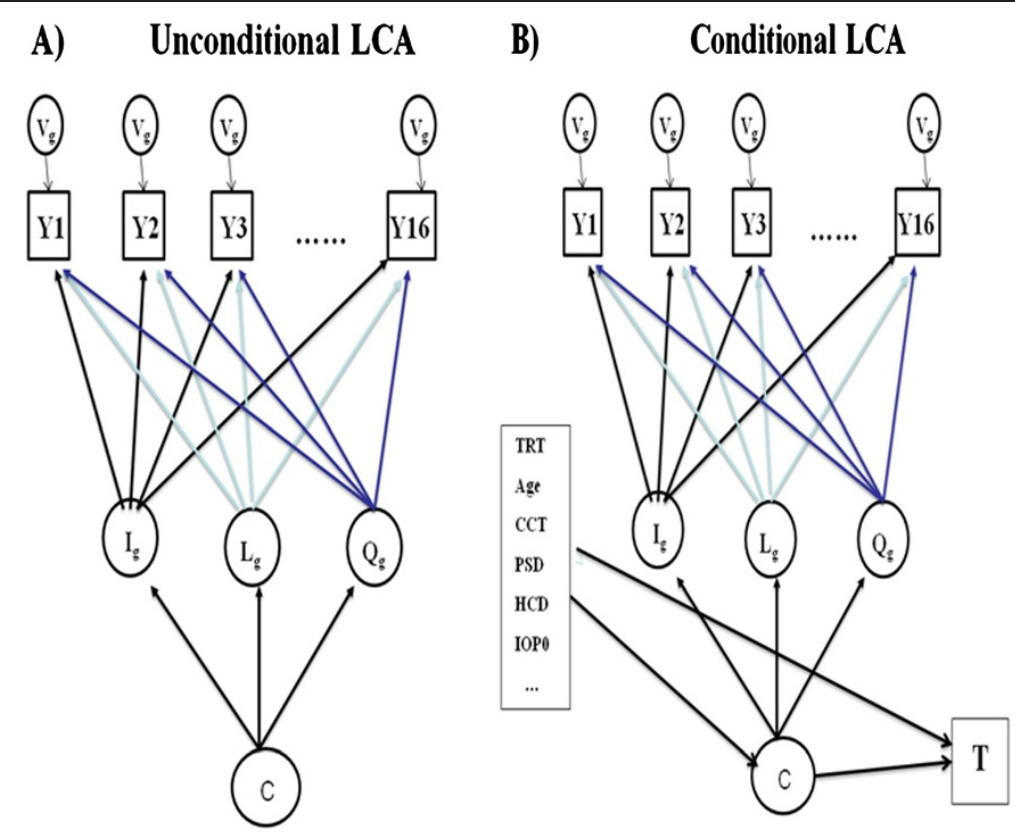

Figure 1 Diagrams for unconditional (1A) and conditional (1B) latent class analysis (LCA) for OHTS data, where C denoted the latent classes. The trajectory of post-randomization IOP $(Y)$ in each class was described by 4 class-specific parameters: the initial IOP level (I), the systematic linear $(\mathrm{L})$ and quadratic $(\mathrm{Q})$ trend over time, and the variance of IOP $(\mathrm{V})$.

values, including the Bayesian Information Criteria (BIC, with a smaller BIC indicating a better fit) and the LoMendell-Rubin adjusted likelihood ratio test (LMR-LRT) [20]. A significant test of LMR-LRT indicated that the model with G-1 classes should be rejected in favor of the G-class LCA. In addition to the above statistical criteria, we also specified a minimum size for each class (with at least 5\% participants in OHTS or 10\% participants in EGPS) to ensure reliable within-class estimation. Once an optimal LCA was developed, a bootstrap method was used to assess whether patients with different patterns of IOP change have different susceptibility to POAG. Specifically, a class membership was generated for each individual from a multinomial distribution using the posterior class probability, and then a Cox model was fit to assess the effects of latent classes on POAG. Summary statistics such as hazard ratios and their $95 \%$ confidence intervals were estimated by repeating the above procedure 1,000 times.

\section{Conditional LCA}

Since patterns of IOP change were found to be associated with the risk of POAG in an unconditional LCA, a conditional LCA was built by adding baseline covariates as predictors to the IOP change and adding time to POAG as an outcome due to IOP change (Figure 1B). Let $\mathrm{X}_{i}$ denote the baseline predictors for $i^{\text {th }}$ subject and $T_{i}=\operatorname{minimum}\left(D_{i}, U_{i}\right)$ be the observed time, where $D_{i}$ was the time to POAG and $\mathrm{U}_{i}$ represented the censoring time independent of $D_{i}$. Let $\Delta_{i}$ be the corresponding event indicator, with $\Delta_{i}=1$ if POAG is observed and $\Delta_{i}=0$ otherwise. Let $\alpha$ and $\beta$ denote effects of baseline covariates $\mathrm{X}_{i}$ on the IOP change and time to POAG respectively. Then the joint distribution of $\left(Y_{i}, T_{i}\right)$ was a mixture distribution defined as [21],

$$
\begin{aligned}
f\left(Y_{i}, T_{i}\right)= & \sum_{g=1}^{G}\left\{\operatorname{Pr}\left(C_{i}=g ; \alpha_{g}\right) \bullet f\left(Y_{i} \mid C_{i}=g ; \theta_{g}\right)\right. \\
& \left.\bullet \lambda\left(T_{i} \mid C_{i}=g ; \beta\right)^{\Delta i} \bullet S\left(T_{i} \mid C_{i}=g ; \beta\right)\right\}
\end{aligned}
$$

- Similar to Model (1), the term $\operatorname{Pr}\left(C_{i}=g ; \alpha_{g}\right)=$ $\frac{\exp \left(\alpha_{0 g}+\alpha_{g} X_{i}\right)}{G}$ represented the size of $g^{\text {th }}$ class $\sum_{h=1}^{G} \exp \left(\alpha_{0 h}+\alpha_{h} X_{i}\right)$ $h=1$
in the mixture distribution and $f\left(Y_{i} \mid C_{i}=g ; \theta_{g}\right)$ described the within-class IOP change.

- The term $\lambda\left(T_{i} \mid C_{i}=g ; \beta\right)=\lambda_{0 g}(\mathrm{t}) \bullet \exp \left(\beta \mathrm{X}_{\mathrm{i}}\right)$ described the risk of developing POAG in $g^{\text {th }}$ class and $S\left(T_{i} \mid C_{i}=g ; \beta\right)=\exp \left(-\int \lambda_{0 g}(\mathrm{t}) \bullet \exp \left(\beta \mathrm{X}_{\mathrm{i}}(\mathrm{dt})\right.\right.$ was the corresponding cumulative POAG-free probability, where $\lambda_{0 \mathrm{~g}}(\mathrm{t})$ was the class-specific baseline hazard with all covariates being 0 . In this paper, $\lambda_{0 \mathrm{~g}}(\mathrm{t})$ was approximated by a piece-wise step-function with a 6-month interval. Following the conventional practice in joint latent class modeling $[21,22]$, we assumed that the association between IOP change and time to POAG was introduced 
exclusively via $\lambda_{0 \mathrm{~g}}(\mathrm{t})$, so that the longitudinal process and survival process were completely independent given the class membership. Therefore, neither time-dependent IOP values nor random effects of IOP were included in the survival function. We also assumed that the effects of covariates on POAG were common across latent classes.

The conditional LCA facilitated a better understanding of ocular hypotensive treatment on the risk of developing POAG. This model allowed us to determine whether the predictive accuracy on POAG can be improved by adding post-randomization IOP. For example, the survival probability (cumulative POAG-free rate) at any time $t$ can be readily calculated as the average of the class-specific survival weighted by the posterior class probabilities,

$$
S\left(T_{i}=t\right)=\sum_{g=1}^{G} \hat{p}_{i g} \bullet \hat{S}\left(T_{i}=t \mid C_{i}=g ; \hat{\beta}\right)
$$

with

$$
\begin{array}{r}
\hat{p}_{i g}=\frac{\widehat{\operatorname{Pr}}\left(C_{i}=g ; \hat{\alpha}_{g}\right) \bullet f\left(Y_{i} \mid C_{i}=g ; \widehat{\theta}_{g}\right)}{\sum_{h=1}^{G}\left\{\widehat{\operatorname{Pr}}\left(C_{i}=h ; \hat{\alpha}_{h}\right) \bullet f\left(Y_{i} \mid C_{i}=h ; \widehat{\theta}_{h}\right)\right\},} \\
\text { and } \hat{S}\left(T_{i}=t \mid C_{i}=g ; \hat{\beta}\right) \\
=\exp \left(-\int_{s=0}^{t} \hat{\lambda}_{0 g}(\mathrm{~s}) \bullet \exp \left(\hat{\beta} \mathrm{X}_{\mathrm{i}}\right) \mathrm{ds}\right),
\end{array}
$$

where $\left(\hat{\theta}_{g}, \hat{\alpha}_{g}, \hat{\beta}\right.$, and $\left.\hat{\lambda}_{0 g}(\mathrm{t})\right)$ were the estimated parameters from the conditional LCA. In this paper, the parameter estimation for LCA was implemented using statistical package Mplus [23], while all the other analyses were performed using statistical package R [24].

\section{Results}

Unconditional LCA

Table 2 showed the fitting statistics of 7 competing LCAs for the OHTS and EGPS data. Based on the model-selection criteria, the IOP change in OHTS was best described by 6 distinct patterns (latent classes), which included $13 \%, 28 \%, 20 \%, 10 \%, 18 \%$ and $11 \%$ of the OHTS subjects respectively. Figure 2 showed the followup IOPs of 50 randomly selected subjects for each class. Most classes were distinguished primarily by the mean IOP levels. The only exceptions were classes 3 and 4 . Classes 3 and 4 had similar average trajectories, but subjects in Class 4 showed a much larger variability. Figure 2 also indicated that the classes with higher mean level and/or larger variability had a higher risk of developing POAG. Table 3 reported the observed frequency of POAG in each class based on the most likely class membership. The hazard ratio (HR) and its $95 \%$ confidence interval (CI) of developing POAG in each class were also calculated using 1000 bootstrapping samples to account for the uncertainty in class membership. The results showed that the last 3 classes had significantly higher risk of developing POAG than the first 3 classes. For reasons that were not clear, however, subjects in Class 2 had the smallest risk though the subjects in Class 1 had the lowest mean follow-up IOP.

In EGPS, the IOP change was best fit by a 5-class LCA (Table 2). Figure 3 showed the post-randomization IOPs of 50 randomly selected subjects from each of the 5 classes, which included 25\%,19\%, 28\%, $16 \%$ and $12 \%$ of EGPS subjects respectively. Subjects in classes 1 and 2 started with similar initial follow-up IOP levels, but those in Class 2 showed a relatively rapid decrease over time. Similarly, subjects in classes 3 and 4 had similar initial levels, but subjects in Class 4 showed a relatively rapid decrease and subjects in Class 3 did not. All subjects in the first 4 classes presented similar magnitude of IOP variability. Subjects in Class 5 had the highest mean

\begin{tabular}{|c|c|c|c|c|c|c|}
\hline \multirow[t]{2}{*}{ \# latent classes (G) } & \multicolumn{3}{|c|}{ OHTS } & \multicolumn{3}{|c|}{ EGPS } \\
\hline & $\overline{B I C}$ & LMR-LRT* & Minimal class size & $\mathrm{BIC}$ & LMR-LRT & Minimal class size \\
\hline 2 & 97097 & $<0.001$ & $47 \%$ & 39235 & $<0.001$ & $44 \%$ \\
\hline 3 & 94219 & 0.002 & $24 \%$ & 38395 & 0.001 & $14 \%$ \\
\hline 4 & 92922 & 0.609 & $14 \%$ & 38109 & 0.005 & $11 \%$ \\
\hline 5 & 92107 & 0.003 & $13 \%$ & 37870 & 0.009 & $12 \%$ \\
\hline 6 & 91644 & 0.042 & $10 \%$ & 37760 & 0.452 & $9 \%$ \\
\hline 7 & 91289 & 0.147 & $7 \%$ & 37682 & 0.060 & $5 \%$ \\
\hline 8 & 91045 & 0.406 & $6 \%$ & 37608 & 0.011 & $4 \%$ \\
\hline
\end{tabular}
level and the largest variability, and they showed a significantly higher risk than the other 4 classes (Table 3 ).

Table 2 Fitting statistics of 7 competing models that are only different in the number of latent classes

* Lo-Mendell-Rubin likelihood ratio test, with a smaller p-value favoring the G-class model over the model with G-1 classes (null hypothesis). 


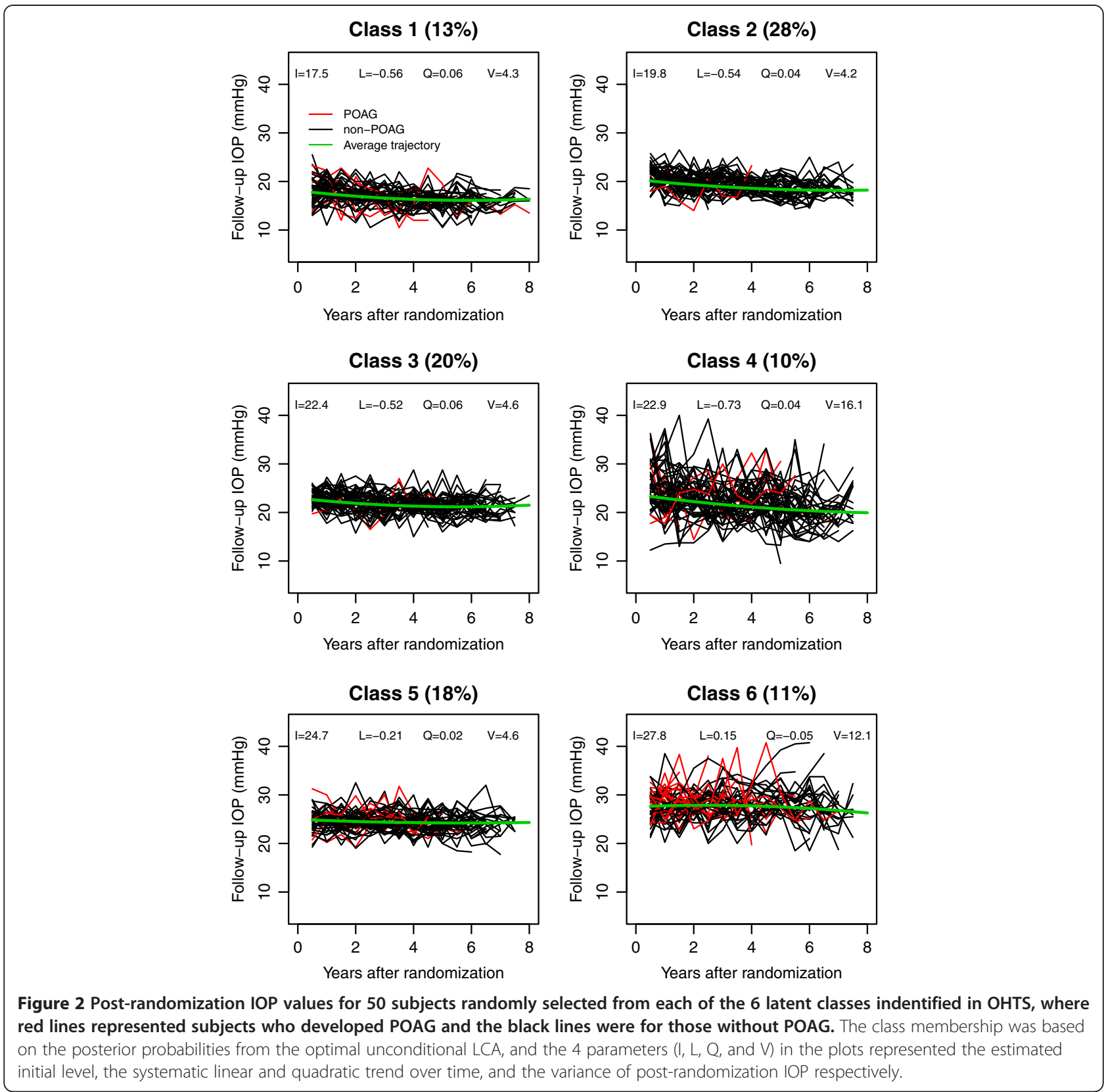

Table 4 presented the distribution of treatment groups across latent classes in the OHTS and EGPS data respectively. In OHTS a great majority of subjects from treatment group fell into the first 3 classes, while in EGPS the distributions of treatment groups were rather similar across all latent classes.

\section{Conditional LCA}

A conditional model was constructed for OHTS and EGPS separately by adding the baseline factors as predictors and the time to POAG as the outcome to the optimal unconditional LCAs (Figure 1B). Since we had an adequate sample size in both studies, no variable- selection procedure was performed and all the baseline covariates (with the exception of dropping the variable race Black from EGPS because of lack of racial diversity) were included as predictors for both IOP change and the risk of developing POAG. Figure 4 presented the modelbased predicted cumulative incidence for an "average" person with all baseline covariates being zero. After controlling for baseline covariates, different patterns of IOP change continued to be prognostic of POAG development. In both studies, the class with the highest mean level was most likely to develop POAG after adjusting for baseline IOP. In OHTS, subjects in Class 4 (with a moderate mean IOP and the largest variability) had 
Table 3 Observed proportions of POAG, as well as estimated hazard ratios (HR) and $95 \%$ confidence intervals $(\mathrm{CI})$ for POAG development in the unconditional LCAs for the OHTS and EGPS data, where the HR and $95 \% \mathrm{Cl}$ were based on 1000 bootstrapping samples to account for the uncertainty in the latent class membership

\begin{tabular}{|c|c|c|c|c|c|c|}
\hline \multirow[t]{2}{*}{ Latent class } & \multicolumn{3}{|c|}{ OHTS } & \multicolumn{3}{|c|}{ EGPS } \\
\hline & POAG\% & HR & $95 \% \mathrm{Cl}$ & POAG\% & HR & $95 \% \mathrm{Cl}$ \\
\hline 1 & $5.9 \%$ & 1.00 & - & $8.3 \%$ & 1.00 & - \\
\hline 2 & $3.9 \%$ & 0.59 & $0.37-0.88$ & $10.2 \%$ & 1.28 & $0.76-2.06$ \\
\hline 3 & $4.3 \%$ & 0.83 & $0.57-1.14$ & $8.7 \%$ & 1.13 & $0.73-1.65$ \\
\hline 4 & $10.1 \%$ & 1.87 & $1.32-2.57$ & $10.5 \%$ & 1.40 & $0.85-2.18$ \\
\hline 5 & $11.4 \%$ & 1.93 & $1.50-2.46$ & $19.4 \%$ & 2.66 & $1.92-3.69$ \\
\hline 6 & $31.2 \%$ & 5.61 & $4.46-7.08$ & & & \\
\hline
\end{tabular}

similar risk as those in Class 5 (with a higher mean IOP and much less variability), but showed a higher risk than those in Class 3 (with the mean IOP comparable to Class 4 but with much less variability). In EGPS, the first 4 classes showed similar risk of developing POAG.

Table 5 presented the estimated parameters for withinclass IOP trajectories, as well as the effects of baseline covariates on IOP classification and the risk of POAG development.

\section{Effects of the baseline covariates on IOP classification}

- To identify baseline predictors for IOP classification, we only focused on factors that were significantly associated with the high risk groups (Classes 4, 5, 6 in OHTS, and Class 5 in EGPS), while treating the lowest risk group (Class 2 in OHTS and Class 1 in EGPS) as the reference. In OHTS, treatment assignment and baseline IOP were two most important predictors for IOP classification. Subjects randomized to treatment group had a much lower chance of inclusion in the high risk groups (with $\mathrm{OR}=0.11,0.003$, and 0.002 for Classes 4, 5, and 6, respectively), while these with a higher baseline IOP were more likely to be in the Classes 4, 5, or 6 (with $\mathrm{OR}=2.80,2.44$, and 5.64 respectively). The results also showed that male subjects were less likely to be in Class $5(\mathrm{OR}=0.51)$, the black subjects were more likely to be in Class $4(\mathrm{OR}=2.12)$ but with a lower chance in Class $5(\mathrm{OR}=0.40)$, and subjects with a history of hypertension were more likely in Class 6 $(\mathrm{OR}=1.93)$. In EGPS, the results confirmed that treatment assignment $(\mathrm{OR}=0.17)$ and baseline IOP level $(\mathrm{OR}=5.99)$ were important predictors for Class 5. The result also showed that older age $(\mathrm{OR}=1.57)$ was significantly associated with Class 5.

\section{Effects of the baseline covariates on the risk of POAG development}

- As expected, the effects of baseline covariates on the risk of developing POAG from the conditional LCA reached consistent conclusions as previous analyses using Cox models $[17,25]$. In OHTS, subjects with older age $(\mathrm{HR}=1.20)$, higher PSD $(\mathrm{HR}=1.26)$, large VCD $(\mathrm{HR}=1.82)$, and history of heart diseases $(\mathrm{HR}=2.03)$ had a higher risk of developing POAG, while thicker CCT $(\mathrm{HR}=0.53)$ and history of diabetes $(\mathrm{HR}=0.19)$ reduced the risk of developing POAG. Interestingly, despite marked differences between OHTS and EGPS in the patterns of IOP change, the EGPS confirmed 4 of the 6 predictors (except age and history of diabetes) identified in OHTS. In both studies, baseline IOP and treatment assignment were not significantly associated with POAG directly, but appeared to affect the risk indirectly through their strong influence on the classification of IOP change.

To explore the effect of follow-up IOP on the overall predictive accuracy of POAG, the 5-year cumulative POAG incidence was calculated for each individual using the formula (3). The overall predictive accuracy was summarized as C-index and calibration statistics (Model 1 in Table 6) [26]. For comparison, Table 6 also presented the C-index and calibration statistics from Cox models that only incorporated baseline predictors (Model 0). The results showed that adding postrandomization IOP considerably improved the predictive accuracy on POAG. In OHTS, for example, C-index increased from 0.778 to 0.821 by adding follow-up IOP. Given the fact that C-index from the baseline model was already high and there was little room for improvement, such an increase was substantial. An improvement in the C-index was also observed in EGPS though in a much smaller magnitude (from 0.706 to 0.719 ). The calibration statistics indicated that the model-based and observed survival probabilities were well agreed in both $\operatorname{OHTS}\left(X^{2}=11.3\right)$ and EGPS $\left(X^{2}=7.0\right)$.

\section{Sensitivity analyses}

As in all the statistical models, LCAs were inevitably based on certain assumptions. One assumption of our LCA was that the trajectories of IOP followed a quadratic functional form. It is known that the parameter estimates, class sizes, and interpretation of latent classes could be heavily influenced by the within-class distribution of longitudinal data [16]. In this section, first we assessed the sensitivity of risk prediction to different LCA specifications. Table 6 presented the $\mathrm{C}$-index and calibration statistics for LCAs after removing the 


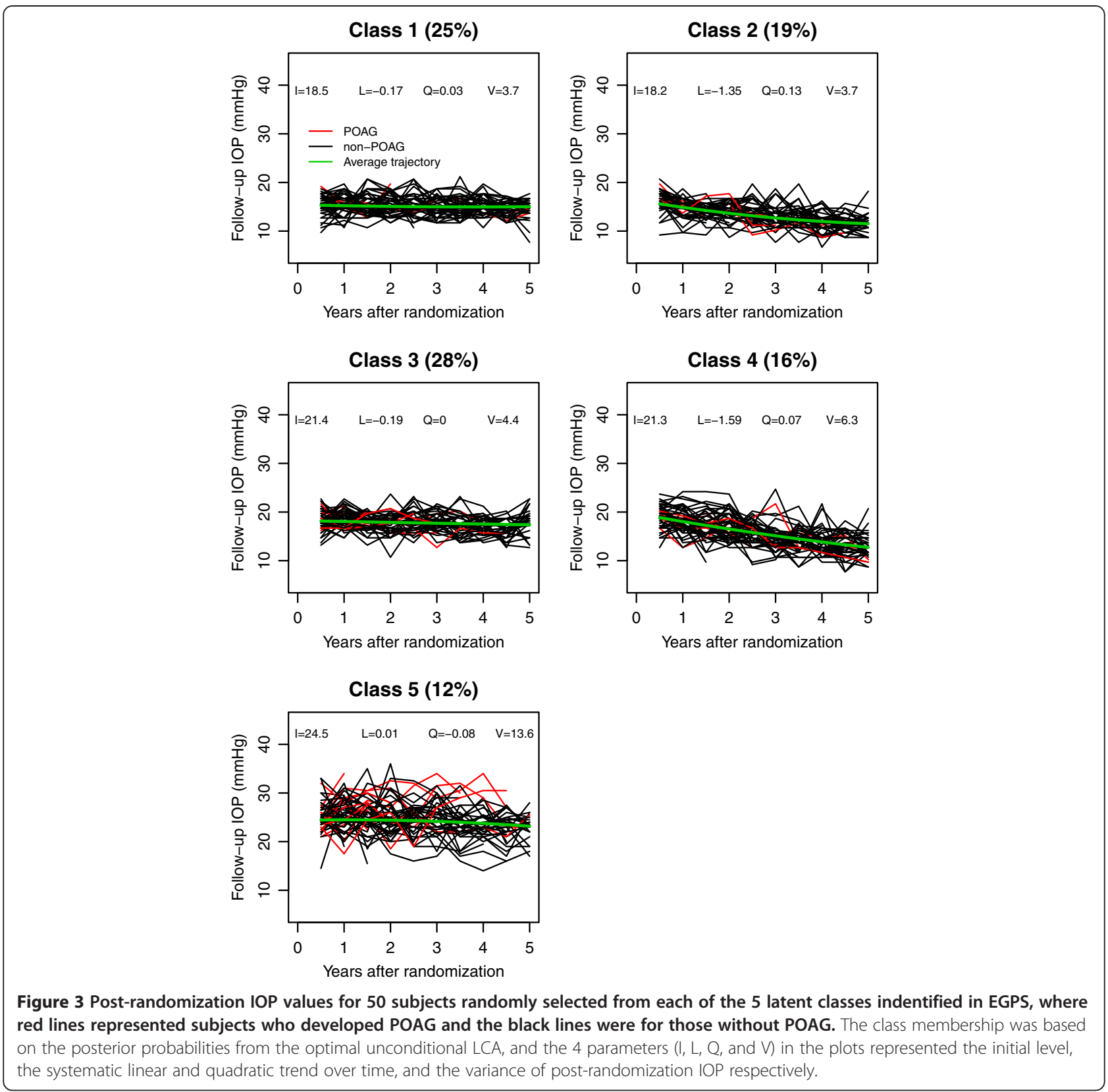

Table 4 Distribution of the randomization groups across latent classes, where the latent classes were based on the most likely posterior class probability from the optimal unconditional LCAs for the OHTS and EGPS data

\begin{tabular}{|c|c|c|c|c|}
\hline \multirow[t]{2}{*}{ Latent class } & \multicolumn{2}{|c|}{ OHTS } & \multicolumn{2}{|c|}{ EGPS } \\
\hline & Observation & Treatment & Placebo & Treatment \\
\hline 1 & 15 (1.9\%) & 191 (24.0\%) & $113(23.3 \%)$ & $143(29.4 \%)$ \\
\hline 2 & $67(8.3 \%)$ & 385 (48.4\%) & 69 (14.3\%) & 112 (23.0\%) \\
\hline 3 & $226(28.1 \%)$ & $106(13.3 \%)$ & $162(33.5 \%)$ & $136(27.9 \%)$ \\
\hline 4 & $55(6.8 \%)$ & $84(10.6 \%)$ & 64 (13.2\%) & 63 (12.9\%) \\
\hline 5 & 279 (34.7\%) & 19 (2.4\%) & 76 (15.7\%) & $33(6.8 \%)$ \\
\hline 6 & $163(20.2 \%)$ & $10(1.3 \%)$ & & \\
\hline Total & 805 (100\%) & 795 (100\%) & 484 (100\%) & 487 (100\%) \\
\hline
\end{tabular}




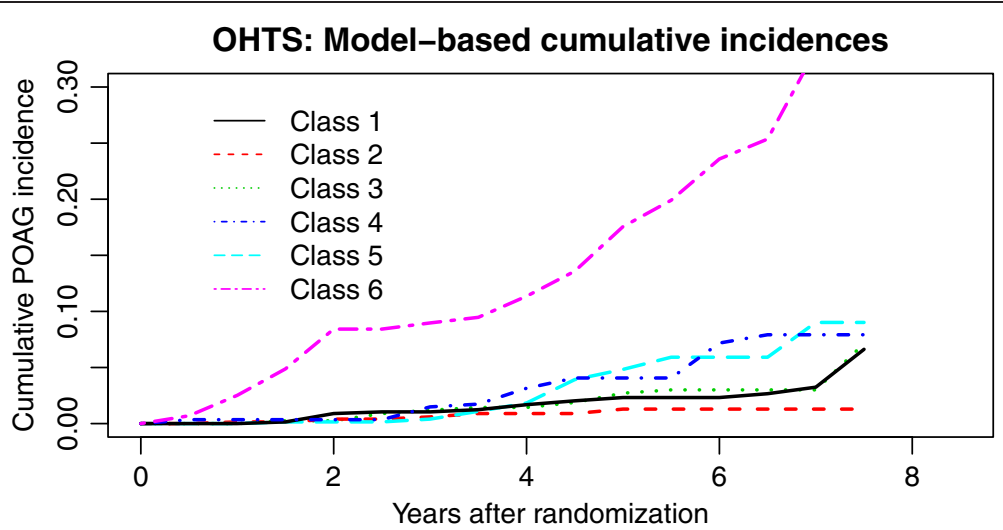

EGPS: Model-based cumulative incidences

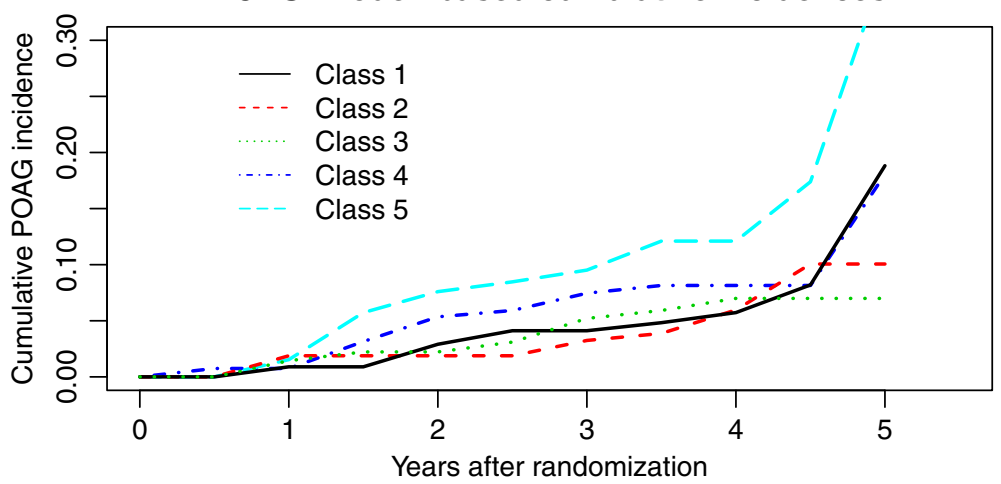

Figure 4 Predicted baseline cumulative incidence of POAG for each class, based on the conditional latent class analysis for the OHTS and EGPS data respectively.

quadratic term (Model 2) or removing both quadratic and linear terms (Model 3). The results showed that LCAs had a robust performance in terms of predictive accuracy for POAG development.

Next, two additional sensitivity analyses were performed in the OHTS data, one excluding participants with Black race and the other only using participants randomized to the observation group. The IOP change in the non-Black was best described by 6 distinct classes, while the LCA in the untreated participants identified 5 classes. Figures $5 \mathrm{~A}$ and $5 \mathrm{C}$ showed the observed mean IOP of latent classes in the non-Black and untreated participants, respectively. Although most classes were distinguished primarily by the mean IOP, each LCA identified a subgroup of participants (Class 4) who had a moderate IOP mean but with the highest IOP variability. More interestingly, the participants from Class 4 in both LCAs showed relatively higher risk of POAG development than those with a comparable mean IOP (Figures 5B and 5D).

Finally, our LCA also made an implicit assumption that the baseline covariates influenced the IOP change exclusively through their effects on the class membership (i.e., no direct effects on the within-class growth parameters). The validity of this assumption can be checked by comparing the conditional LCAs with the unconditional models. The assumption violation is often signified by a dramatic shifting in the meaning and size of latent classes when the baseline predictors are added to the unconditional LCA [16]. Based on the estimated class-specific parameters (Table 5, Figures 2 and 3), this assumption was well satisfied in both studies.

\section{Discussion}

In recent years, one of the hot topics in glaucoma research has been the effect of IOP fluctuation on POAG. Although more and more studies have confirmed that a decrease in the mean IOP level can reduce the risk of developing POAG, the findings from major prospective clinical trials about the impact of IOP fluctuation on POAG remain controversial [25,27-30]. In this paper, we analyzed the post-randomization IOPs from OHTS and EGPS taking a latent class analysis (LCA) approach. The LCA allows us to identify distinct patterns of IOP change over time and then associates the changes in IOP with the risk of POAG. The results from both studies showed that different patterns of IOP change could markedly affect the risk of POAG (irrespective of their baseline, pre-randomization IOP levels). In OHTS, the 
Table 5 Estimated parameters of the conditional LCAs in the OHTS and EGPS data OHTS

\begin{tabular}{|c|c|c|c|c|c|c|c|}
\hline \multirow[t]{2}{*}{ Variables } & \multicolumn{6}{|c|}{ Parameters for IOP change and the effects of covariates on class membership } & \multirow{2}{*}{$\begin{array}{l}\text { Effects on } \\
\text { POAG }\end{array}$} \\
\hline & Class 1 & Class2 (Ref.) & Class 3 & Class 4 & Class 5 & Class 6 & \\
\hline $\begin{array}{l}\text { Class Size } \\
\text { IOP Change }\end{array}$ & $14.2 \%$ & $27.1 \%$ & $21.1 \%$ & $9.1 \%$ & $17.6 \%$ & $10.9 \%$ & \\
\hline I & $17.58(0.20)^{\#}$ & $19.83(0.21)^{\#}$ & $22.30(0.21)^{\#}$ & $22.82(0.79)^{\#}$ & $24.74(0.22) \#$ & $27.70(0.28)^{\#}$ & - \\
\hline L & $-0.57(0.08)^{\#}$ & $-0.53(0.06)^{\#}$ & $-0.47(0.09)^{\#}$ & $-0.95(0.30)^{\#}$ & $-0.20(0.09)^{*}$ & $0.16(0.17)$ & - \\
\hline Q & $0.06(0.01)^{\#}$ & $0.05(0.01)^{\#}$ & $0.05(0.01)^{\#}$ & $0.07(0.04)$ & $0.02(0.02)$ & $-0.05(0.03)$ & - \\
\hline $\mathrm{V}$ & $4.36(0.27)^{\#}$ & $4.30(0.32)^{\#}$ & $4.80(0.25)^{\#}$ & $16.07(1.46)^{\#}$ & 4.66(0.31)\# & $12.15(1.15)^{\#}$ & - \\
\hline \multicolumn{8}{|l|}{ Covariates } \\
\hline Intercept & $-2.64(0.63)^{\#}$ & & $2.08(0.47)^{\#}$ & $-0.06(0.47)$ & $2.35(0.54)^{\#}$ & $1.04(0.61)$ & - \\
\hline $\begin{array}{l}\text { TRT } \\
\end{array}$ & $1.60(0.66)^{*}$ & - & $-3.25(0.35)^{\#}$ & $-2.19(0.63)^{\#}$ & $-5.98(0.56)^{\#}$ & $-6.46(0.70)^{\#}$ & $0.16(0.29)$ \\
\hline MALE & $0.25(0.23)$ & - & $-0.99(0.24)^{\#}$ & $0.24(0.27)$ & $-0.68(0.28)^{*}$ & $-0.22(0.30)$ & $0.23(0.19)$ \\
\hline RACEB & $-0.10(0.27)$ & - & $-0.37(0.30)$ & $0.75(0.31)^{*}$ & $-0.91(0.34)^{*}$ & $0.05(0.37)$ & $-0.05(0.24)$ \\
\hline AGE & $0.06(0.12)$ & - & $-0.01(0.12)$ & $0.08(0.16)$ & $-0.05(0.14)$ & $0.13(0.15)$ & $0.18(0.09)^{*}$ \\
\hline IOPO & $-0.79(0.18)^{\#}$ & - & $0.21(0.22)$ & $1.03(0.36)^{*}$ & $0.89(0.18)^{\#}$ & $1.73(0.22)^{\#}$ & $-0.10(0.11)$ \\
\hline $\mathrm{CCT}$ & $-0.35(0.12)^{*}$ & - & $0.18(0.11)$ & $-0.08(0.17)$ & $0.14(0.13)$ & $0.09(0.16)$ & $-0.64(0.13)^{\#}$ \\
\hline PSD & $0.13(0.11)$ & - & $0.04(0.11)$ & $0.08(0.13)$ & $0.17(0.13)$ & $0.12(0.15)$ & $0.23(0.10)^{*}$ \\
\hline VCD & $0.06(0.11)$ & - & $-0.08(0.12)$ & $-0.15(0.17)$ & $-0.10(0.13)$ & $-0.09(0.15)$ & $0.60(0.10)^{\#}$ \\
\hline $\mathrm{BB}$ & $-0.60(0.48)$ & - & $-0.37(0.61)$ & $-{ }^{* *}$ & $-0.30(0.62)$ & $-1.21(0.77)$ & $0.19(0.57)$ \\
\hline $\mathrm{CHB}$ & $-0.19(0.37)$ & - & $-0.32(0.42)$ & $0.47(0.45)$ & $-0.42(0.44)$ & $-0.50(0.49)$ & $0.09(0.31)$ \\
\hline DM & $-0.23(0.35)$ & - & $0.22(0.32)$ & $-0.71(0.45)$ & $0.23(0.36)$ & $0.64(0.40)$ & $-1.67(0.53)^{*}$ \\
\hline HEART & $0.70(0.44)$ & - & $0.10(0.49)$ & $0.43(0.56)$ & $-0.09(0.56)$ & $-0.48(0.73)$ & $0.71(0.29)^{*}$ \\
\hline $\mathrm{HBP}$ & $0.47(0.24)^{*}$ & - & $0.40(0.27)$ & $0.06(0.34)$ & $0.41(0.30)$ & $0.66(0.33)^{*}$ & $0.08(0.22)$ \\
\hline \multicolumn{8}{|c|}{ EGPS } \\
\hline \multirow[t]{2}{*}{ Variables } & \multicolumn{5}{|c|}{ Parameters for IOP change and the effects of covariates on class membership } & \multirow[t]{2}{*}{ Effects on POAG } & \\
\hline & Class1 (Ref.) & Class 2 & Class 3 & Class 4 & Class 5 & & \\
\hline $\begin{array}{l}\text { Class Size } \\
\text { IOP Change }\end{array}$ & $26.3 \%$ & $20.1 \%$ & $29.3 \%$ & $12.9 \%$ & $11.4 \%$ & & \\
\hline I & $18.66(0.25)^{\#}$ & $18.24(0.18)^{\#}$ & $21.24(0.20)^{\#}$ & $21.85(0.47)^{\#}$ & $24.33(0.34)^{\#}$ & - & \\
\hline $\mathrm{L}$ & $-0.34(0.14)^{*}$ & $-1.29(0.18)^{\#}$ & $-0.25(0.11)^{*}$ & $-1.76(0.28)^{\#}$ & $0.06(0.26)$ & - & \\
\hline Q & $0.05(0.03)$ & $0.11(0.04)^{*}$ & $0.02(0.03)$ & $0.02(0.07)$ & $-0.08(0.07)$ & - & \\
\hline V & $3.79(0.23)^{\#}$ & $3.75(0.25)^{\#}$ & $4.59(0.24)^{\#}$ & $7.12(0.85)^{\#}$ & $12.17(1.32)^{\#}$ & - & \\
\hline \multicolumn{8}{|l|}{ Covariates } \\
\hline Intercept & - & $-0.84(0.33)^{*}$ & $0.30(0.28)$ & $-0.85(0.58)$ & $-0.71(0.34)^{*}$ & - & \\
\hline TRT & - & $0.36(0.27)$ & $-0.65(0.23)^{*}$ & $-0.58(0.37)$ & $-1.79(0.37)^{\#}$ & $-0.01(0.21)$ & \\
\hline MALE & - & $-0.35(0.29)$ & $0.14(0.23)$ & $0.18(0.40)$ & $0.38(0.33)$ & $-0.24(0.22)$ & \\
\hline Black & - & - & - & - & - & - & \\
\hline AGE & - & $-0.09(0.16)$ & $0.01(0.13)$ & $0.40(0.23)$ & $0.45(0.20)^{*}$ & $0.16(0.10)$ & \\
\hline IOPO & - & $-0.61(0.24)^{*}$ & $0.82(0.17)^{\#}$ & $1.24(0.24)^{\#}$ & $1.79(0.23)^{\#}$ & $0.11(0.13)$ & \\
\hline $\mathrm{CCT}$ & - & $-0.33(0.13)^{*}$ & $-0.14(0.12)$ & $-0.43(0.15)^{*}$ & $0.09(0.15)$ & $-0.36(0.12)^{*}$ & \\
\hline PSD & - & $0.27(0.16)$ & $-0.23(0.14)$ & $-0.18(0.24)$ & $-0.41(0.23)$ & $0.18(0.09)^{*}$ & \\
\hline VCD & - & $-0.13(0.17)$ & $-0.03(0.13)$ & $0.72(0.26)^{*}$ & $0.17(0.16)$ & $0.46(0.12)^{\#}$ & \\
\hline $\mathrm{BB}$ & - & $-0.17(0.50)$ & $-0.82(0.52)$ & $-{ }^{* *}$ & $-0.58(0.69)$ & $-0.07(0.41)$ & \\
\hline $\mathrm{CHB}$ & - & $-0.10(0.56)$ & $-0.97(0.52)$ & $0.89(1.03)$ & $-1.22(0.79)$ & $-0.28(0.47)$ & \\
\hline DM & - & $-0.46(0.52)$ & $0.12(0.45)$ & $-1.32(1.27)$ & $0.82(0.81)$ & $-0.18(0.54)$ & \\
\hline HEART & - & $0.78(0.41)$ & $0.11(0.44)$ & $-0.83(0.77)$ & $-0.79(0.61)$ & $0.74(0.32)^{*}$ & \\
\hline $\mathrm{HBP}$ & - & $0.02(0.36)$ & $0.53(0.30)$ & $-1.27(0.90)$ & $0.11(0.54)$ & $0.24(0.26)$ & \\
\hline
\end{tabular}

${ }^{*}: p<0.05 ; \#: p<0.001 ; *^{* *}$ : Not estimable due to zero count of beta blocker use in the given class. 
Table 6 Sensitivity analysis comparing the overall predictive accuracy (measured as C-index and Calibration Chi-square statistics) for LCAs with different model specifications

\begin{tabular}{llllcr}
\hline Model & Model Features & \multicolumn{2}{c}{ C index } & \multicolumn{2}{c}{ Calibration Chi-square } \\
\cline { 3 - 6 } & & OHTS & EGPS & OHTS & EGPS \\
\hline 0 & Cox mode with baseline factors only & 0.778 & 0.706 & 5.0 & 2.1 \\
1 & LCA with a quadratic within-class functional form & 0.821 & 0.719 & 11.3 & 7.0 \\
2 & LCA with a linear within-class functional form & 0.825 & 0.720 & 10.2 & 10.5 \\
3 & LCA with a constant within-class functional form & 0.823 & 0.727 & 4.9 & 13.5 \\
\hline
\end{tabular}

change in IOP was best described by 6 distinct patterns. The model identified a subset of participants in whom IOP variability also played an important role in predicting POAG. This subgroup showed the highest IOP variability and had a higher risk than those with a comparable IOP mean. Comparing to the reference class, these participants were less likely from treatment group $(\mathrm{OR}=0.11)$, more likely self-classified as being black $(\mathrm{OR}=2.12)$, and had relatively higher baseline IOP $(\mathrm{OR}=2.80)$. However, the subgroup only accounted for about $10 \%$ of the OHTS sample, and this may partially explain our finding that IOP variability was an independent risk factor in the OHTS but had little impact on the overall predictive accuracy for POAG (manuscript in progression). In a sensitivity analysis using the non-Black participants, the LCA identified similar patterns of IOP change as in the whole OHTS dataset. This result was consistent with a tree-based model in the OHTS-EGPS meta-analysis which showed that race was no longer an important predictor for POAG development after considering other risk factors [17]. In EGPS, LCA identified 5 distinct latent classes and confirmed that those subjects with the highest mean IOP were most likely to develop POAG. However, it failed to disentangle the effect of fluctuation from mean because these participants with the highest mean level also had

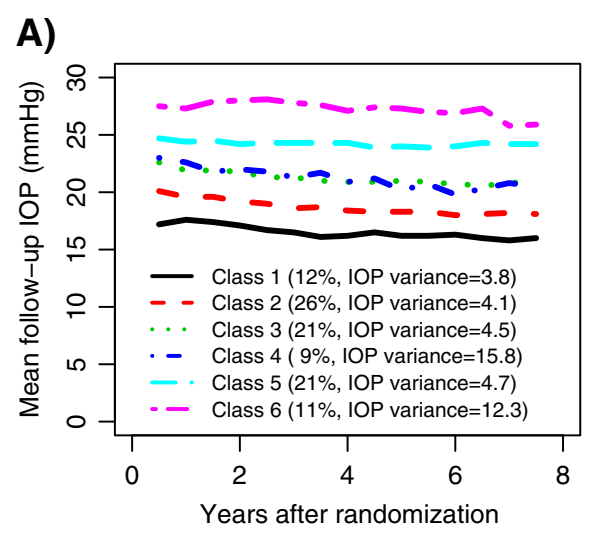

B)

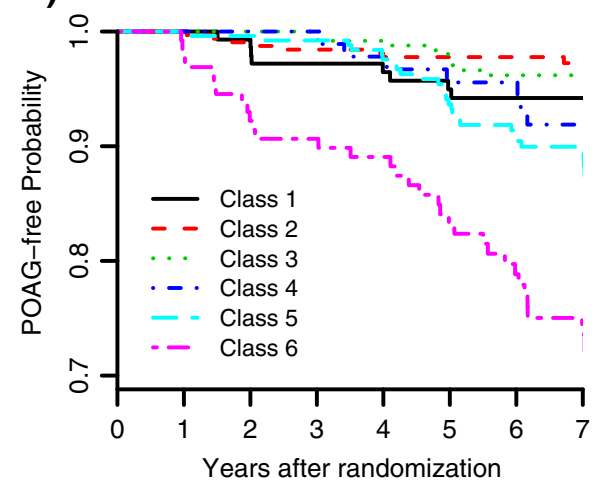

C)

D)
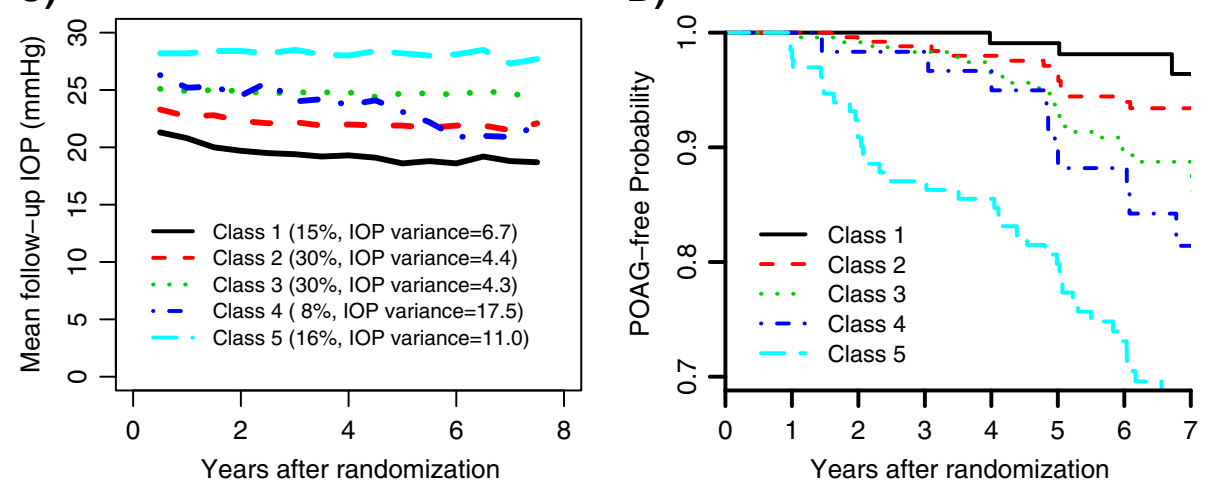

Figure 5 Sensitivity analyses of latent class models in the OHTS data. Plots $\mathbf{A}$ and $\mathbf{B}$ : the observed mean IOP during follow-up visits of the latent classes and the corresponding Kaplan-Meier POAG-free curves in the non-Black participants; Plots C and D: the observed mean IOP during follow-up visits of the latent classes and the corresponding Kaplan-Meier POAG-free curves in the untreated participants. 
the largest IOP variability. Interestingly, despite the marked differences between EGPS and OHTS in the treatment intervention and magnitude of IOP lowering achieved, both studies showed that adding IOP change into the baseline model improved the overall predictive accuracy for POAG development.

Conventionally the change of longitudinal data is described using linear mixed models with random coefficients [31]. Though the mixed model recognizes the heterogeneous nature of the data by allowing each individual to have his/her own intercept and slope, it assumes that all individuals come from a single population and uses an average trajectory for the entire population. A LCA analyzes data from a rather different perspective. The model approximates the unknown heterogeneity in the distribution of longitudinal outcome using a finite number of polynomial functions each describing a unique subpopulation [14,32]. It classifies individuals into distinct groups based on the patterns of longitudinal outcome, so that individual within a group are more similar than those between different groups. This LCA possesses some unique advantages as comparing to conventional methods. First, the model lends itself directly to a set of well characterized subpopulations and also provides a formal statistical procedure to determine the appropriate number of subpopulations. It thus enables the discovery of unexpected yet potentially meaningful subpopulations that may be otherwise missed with conventional methods. Second, the method permits one to relate the developmental patterns of longitudinal data to its antecedents (predictors or covariates) and consequences (clinical outcomes), and thus allows estimation of both direct and indirect (via longitudinal data) effects of a covariate on the distant outcome $[16,23]$. Finally, the recent advances of the dual trajectory modeling also allow investigators to assess the joint evolution of multiple longitudinal processes, which may evolve contemporaneously or over different time periods [32].

LCA also provides an attractive alternative for making prediction with time-dependent covariates [21,22]. A LCA takes a joint modeling approach to assess the association between longitudinal and survival data and thus uses information more efficiently, resulting less biased estimates. Unlike the conventional joint models that assess the association via shared random effects [19,33,34], a LCA relates the longitudinal process to survival process by latent classes and assumes the two stochastic processes independent given the class membership [22]. Therefore, neither time-dependent covariates nor random effects of the longitudinal data are needed in the survival sub-model. Such a model specification will avoid the intensive computation to obtain the random effects for new subjects and hence facilitates a real-time individualized prediction [21]. The key to build an accurate prediction in a LCA setting is to have a reliable classification given the observed data. Generally speaking, the more the available serial biomarker readings, the more reliable a classification is. To this consideration, the impact of follow-up IOP on POAG may be overestimated in OHTS because an average length of 6.5-year IOP readings was used to calculate the 5-year POAG-free rate. To solve this dilemma, which is rather common in all predictions involving time-dependent covariates, one of the most frequently used approaches in medical literature is a landmark analysis that consists of fitting a serial of survival models only to the subjects still at risk, that is, computation of the predictive distribution at a certain time given the history of event and covariates until that moment [35]. In a LCA setting, such a dynamic prediction can be conveniently implemented because the conditional survival probability at any time can be calculated analytically from a single LCA once the parameters are estimated [21].

Despite its advantages, the LCA has several limitations. First, the computational load of LCA can be high, especially for models with complexity structures. In OHTS data $(\mathrm{N}=1600)$, for example, it ran less than 10 minutes for an unconditional 6-class LCA, but it took more than 30 minutes to develop the full conditional model. Because of the exploratory nature of data analysis with LCA, the cumulative time can be substantial. For this consideration, in practice the best LCA model is often constructed taking a two-step approach as in this paper. Another issue in LCA is that the log-likelihood function may end up at local rather than global maxima. Fortunately this issue has been taken into consideration by the statistical package Mplus which automatically uses 10 sets of randomly generated starting values for estimation. The program also allows investigators to rerun and compare the estimates from user specified starting values if necessary [23].

\section{Conclusion}

LCA provides a useful alternative to understand the interrelationship among the baseline covariates, the change in follow-up IOP, and the risk of developing POAG. The inclusion of post-randomization IOP can improve the predictive ability of the original prediction model that only included baseline risk factors.

\footnotetext{
Abbreviations

IOP: Intraocular pressure; POAG: Primary open-angle glaucoma; LCA: Latent class analysis; OHTS: The ocular hypertension treatment study; EGPS: The european glaucoma prevention study; HR: Hazards ratio; OR: Odds ratio; CCT: Central corneal thickness; PSD: Pattern standard deviation; VCD: Vertical cup/disc ratio
}

Competing interests

The authors declare that they have no competing interests. 


\section{Authors' contributions}

FG, JPM, JAB and MOG conceived the study. FG and JAB carried out the data analysis. FG and MOG drafted the first version of the manuscript. All authors contributed to the critical review and approved the final version.

\section{Acknowledgments}

This study is supported by grants from the National Eye Institute of Health (EY091369 and EY09341) and Research to Prevent Blindness (RPB).

\section{Author details}

'Division of Biostatistics, Washington University School of Medicine, St. Louis, MO 63110, USA. ${ }^{2}$ Department of Ophthalmology \& Visual Sciences,

Washington University School of Medicine, St. Louis, MO 63110, USA. ${ }^{3}$ Department of Ophthalmology, The Policlinico di Monza, University Bicocca of Milan, Milan, Italy. ${ }^{4}$ Laboratory of New Drugs Development Strategies, Mario Negri Institute, Milan, Italy.

Received: 21 October 2011 Accepted: 25 September 2012 Published: 4 October 2012

\section{References}

1. Quigley HA: Number of people with glaucoma worldwide. $\mathrm{Br}$ J Ophthalmol 1996, 80(5):389-393.

2. Sommer A, Tielsch JM, Katz J, et al: Racial differences in the cause-specific prevalence of blindness in east Baltimore. N Engl J Med 1991, 325(20):1412-1417.

3. US Department of Health, Education, and Welfare: Statistics on blindness in the model reporting area 1969-1970. Washington, DC: US Government Printing Office; 1973

4. Quigley HA, Vitale S: Models of open-angle glaucoma prevalence and incidence in the United States. Invest Ophthalmol Vis Sci 1997. 38(1):83-91.

5. Hyman L, Wu SY, Connell AM, et al: Prevalence and causes of visual impairment in the Barbados eye study. Ophthalmology 2001, 108(10):1751-1756.

6. Leibowitz HM, Krueger DE, Maunder LR, et al: The Framingham Eye study monograph: an ophthalmological and epidemiological study of cataract, glaucoma, diabetic retinopathy, macular degeneration, and visual acuity in a general population of 2631 adults, 1973-1975. Surv Ophthalmol 1980, 24(suppl):335-610

7. Armaly MF, Kreuger DE, Maunder $L$, et al: Biostatistical analysis of the collaborative glaucoma study. I. Summary report of the risk factors for glaucomatous visual-field defects. Arch Ophthalmol 1980, 98:2163-2171.

8. Quigley HA, Enger C, Katz J, et al: Risk factors for the development of glaucomatous visual field loss in ocular hypertension. Arch Ophthalmol 1994, 112:644-649.

9. Kass MA, Heuer DK, Higginbotham EJ, et al: The ocular hypertension treatment study: a randomized trial determines that topical ocular hypotensive medication delays or prevents the onset of primary open-angle glaucoma. Arch Ophthalmol 2002, 120(6):701-713. discussion 829-830.

10. Heijl A, Leske MC, Bengtsson B, et al: Reduction of intraocular pressure and glaucoma progression: results from the early manifest glaucoma trial. Arch Ophthalmol 2002, 120(10):1268-1279.

11. The Advanced Glaucoma Intervention Study Group: The advanced glaucoma intervention study (AGIS): 7 . The relationship between control of intraocular pressure and visual field deterioration. Am J Ophthalmo 2000, 130:429-440.

12. Singh K, Shrivastava A: Intraocular pressure fluctuations: how much do they matter? Curr Opin Ophthalmol 2009, 20:84-87.

13. Sultan MB, Mansberger SL, Lee PP: Understanding the importance of IOP variables in glaucoma: a systematic review. Surv Ophthalmol 2009, 54:643-662.

14. Nagin DS: Group-based modeling of development. Cambridge: MA. Harvard University Press; 2005.

15. Miglior S, Pfeiffer N, Torri V, Zeyen T, Cunha-Vaz J, Adamsons I: Predictive factors for open-angle glaucoma among subjects with ocular hypertension in the European glaucoma prevention study. Ophthalmology 2007, 114(1):3-9.

16. Petras H, Masyn K: General growth mixture analysis with antecedents and consequences of change. Springer: Handbook of Quantitative Criminology; 2009.
17. Gordon MO, Torri V, Miglior S, Beiser JA, Floriani I, Miller JP, Gao F, Adamsons I, Poli D, D'Agostino RB, Kass MA: The ocular hypertension treatment study group and the European glaucoma prevention study group. A validated prediction model for the development of primary open angle glaucoma in individuals with ocular hypertension. Ophthalmology 2007, 114(1):10-19.

18. Gordon MO, Beiser JA, Brandt JD, Heuer D, Higginbotham E, Johnson C, et al: The ocular hypertension treatment study: baseline factors that predict the onset of primary open-angle glaucoma. Arch Ophthalmol 2002, 120:714-720.

19. Gao F, Miller JP, Miglior S, Beiser JA, Torri V, Kass MA, Gordon MO: A joint model for prognostic effect of biomarker variability on outcomes: long-term intraocular pressure (IOP) fluctuation on the risk of developing primary open-angle glaucoma (POAG). JP Journal of Biostatistics. 2011, 5(2):73-96.

20. Lo Y, Mendell NR, Rubin DB: Testing the number of components in a normal mixture. Biometrika 2001, 88:767-778.

21. Proust-Lima C, Taylor JMG: Development and validation of a dynamic prognostic tool for prostate cancer recurrence using repeated measures of posttreatment PSA: a joint modeling approach. Biostatistics 2009, 10:535-549.

22. Lin H, Turnbull BW, Mcculloch CE, Slate EH: Latent class models for joint analysis of longitudinal biomarker and event process data: application to longitudinal prostate-specific antigen readings and prostate cancer. J Am Stat Assoc 2002, 97:53-65

23. Muthén LK, Muthén BO: Mplus User's quide. Sixthth edition. Los Angeles, CA: Muthén \& Muthén; 1998-2010.

24. R Core Team: $R$ : a language and environment for statistical computing. Vienna, Austria: R Foundation for Statistical Computing; 2012. http://www.R-project.org/. ISBN 3-900051-07-0,

25. Miglior S, Torri V, Zeyen T, Pfeiffer N, Cunha-Vaz J, Adamsons I: Intercurrent factors associated with the development of open-angle glaucoma in the European glaucoma prevention study. Am J Ophthalmol 2007, 144:266-275.

26. Harrell FE Jr, Lee KL, Mark DB: Tutorial in biostatistics multivariate prognostic models: Issues in developing models, evaluation assumptions and adequacy, and measuring and reducing errors. Stat Med 1996, 15:361-387

27. Nouri-Mahdavi K, Hoffman D, Coleman AL, et al: Advanced glaucoma intervention study. Predictive factors for glaucomatous visual field progression in the advanced glaucoma intervention study. Ophthalmology 2004, 111(9):1627-1635.

28. Bengtsson BL, Leske MC, Hyman L, Heijl A, Early Manifest Glaucoma Trial Group: Fluctuation of intraocular pressure and glaucoma progression in the early manifest glaucoma trial. Ophthalmology 2007, 114:205-209.

29. Caprioli J, Coleman AL: Intraocular pressure fluctuation: a risk factor for visual field progression at low intraocular pressures in the advanced glaucoma intervention study. Ophthalmology 2008, 115:1123-1129.

30. Medeiros FA, Weinreb RN, Zangwill LM, et al: Long-term intraocular pressure fluctuations and risk of conversion from ocular hypertension to glaucoma. Ophthalmology 2008, 115:934-940

31. Laird NM, Ware JH: Random-effects models for longitudinal data. Biometrics 1982, 38:963-974.

32. Jones BL, Nagin DS: Advances in group-based trajectory modeling and a SAS procedure for estimating them. Sociological Methods \& Research. 2007, 35(3):542-571.

33. Henderson R, Diggle P, Dobson A: Joint modeling of longitudinal measurements and event time data. Biostatistics 2000, 4:465-480

34. Vonesh EF, Greene T, Schluchter MD: Shared parameter models for the joint analysis of longitudinal data and event times. Statistics in Medicine 2006, 25:143-163.

35. Van Houwelingen HC: Dynamic prediction by landmarking in event history analysis. Scand J Statist 2007, 34:70-85.

doi:10.1186/1471-2288-12-151

Cite this article as: Gao et al:: The effect of changes in intraocular pressure on the risk of primary open-angle glaucoma in patients with ocular hypertension: an application of latent class analysis. BMC Medical Research Methodology 2012 12:151. 